\section{Tropical Journal of Pathology and Microbiology}

2021 Volume 7 Number 4 July-August

\title{
Risk factors and microbiological features of surgical site infections
}

\author{
Kumar A. ${ }^{1}$, Sinha S. $^{2 *}$ \\ DOI: https://doi.org/10.17511/jopm.2021.i04.08
}

${ }^{1}$ Atul Kumar, Associate Professor, Department of Surgery, Government Medical College, Ratlam, Madhya Pradesh, India.

2* Shivani Sinha, Demonstrator, Department of Microbiology, Government Medical College, Ratlam, Madhya Pradesh, India.

Background: Surgical site infections are the infections of the tissues, organs or spaces exposed by surgeons during the performance of an invasive procedure manifested in the postoperative period within 30 days after a surgical procedure and up to one year if an implant was placed in the patient. SSI is still higher in India as compared to western data leading to increased morbidity and mortality. This study aimed to analyse the factors associated with SSI and its microflora in a tertiary care centre. Methods: A descriptive cross-sectional study was done for one year in a tertiary care centre in Bareilly, U.P. All the patients who underwent surgeries related to git pathology in the surgery department, either emergency or elective with surgical site infections, were included in this study. Patients less than 18, all cases operated else were, or re-operated were excluded from this study. All traumatic abdominal surgeries were also excluded from this study. Results: The present study revealed SSI was associated with $82(9.4 \%)$ cases among elective and $80(21 \%)$ in emergency surgeries. Superficial incision SSI was found to be most common (90 cases), followed by deep incisional SSI (60 patients) and organ/space SSI (12 cases). Minor intestine surgeries were most commonly associated with SSI. The microbiological organism most widely isolated was E. Coli $(41.2 \%)$, followed by Staphylococcus aureus $(27.5 \%)$, Klebsiella $(16.5 \%)$, group A beta-haemolytic Streptococci $(9.1 \%)$ and Pseudomonas (5.5\%). Conclusions: Surgical site infection still causes considerable morbidity and high cost to the health care system and is becoming increasingly crucial in medico-legal aspects. Prevention of SSI requires a targeted approach to improve health care workers' knowledge, practising the guidelines strictly regarding asepsis and optimising the patient before surgery. Reduction in rate of SSI will improve cosmesis and make the results of operations better as a whole.

Keywords: Nosocomial infection, SSI, Surgical site infection

Corresponding Author

Shivani Sinha, Demonstrator, Department of Microbiology, Government Medical College, Ratlam, Madhya Pradesh, India.

Email: drshivani1705@gmail.com

\section{How to Cite this Article}

Atul Kumar, Shivani Sinha, Risk factors and microbiological features of surgical site infections. Trop J Pathol Microbiol. 2021;7(4):201-206. Available From https://pathology.medresearch.in/index.php/jopm/ar ticle/view/563
To Browse

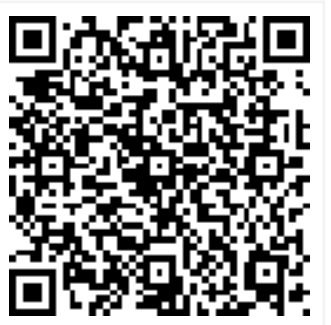

Manuscript Received 2021-07-04

Conflict of Interest No 2021-07-14

Funding

Nil

Review Round 2
2021-07-30
Ethical Approval
Yes

Review Round 2

Yes
Review Round 3 2021-08-12

Plagiarism X-checker $7 \%$
Accepted 2021-08-23

Note 


\section{Introduction}

Surgical site infections are the infections of the tissues, organs, or spaces exposed by surgeons during an invasive procedure manifested in the postoperative period. Due to the wide spectrum of possible clinical and pathological features, it is challenging to define SSI accurately. As per the NNIS program, SSIs are classified into incisional and organ/space infections[1]. Incisional SSI is further subclassified into superficial and deep incisional categories. Superficial incisional SSI occurs within 30 days after the surgery, while deep incisional, organ or space SSI includes infections within 30 days and up to 1 year if the implant is present[1]. The development of SSIs is not only related to the degree of microbial contamination of the wound during surgery but also the duration of the procedure and host factors such as diabetes, malnutrition, obesity, immune suppression, and several other underlying disease states

Surgical site infection still causes considerable morbidity and high cost to the health care system and is becoming increasingly crucial in medico-legal aspects. It is the third most common cause of nosocomial infections and most common among surgical patients complicating nearly one out of four surgeries. While the global estimate of SSI varied from $0.5 \%$ to $15 \%$ in different studies, still in India, it ranges from 23 to $38 \%$ [2]. Even with the advancement in aseptic techniques and standard patient care in the world, lack of optimisation of patient condition, the poor operational environment, inappropriate assessment and lack of appropriate measures leads to a higher rate of SSI in most of the cities. Surgical site infection is an essential factor related to the outcome of the surgery but is also treated as a failure of surgery in this modern era[3]. This study was proposed to find out the associated factors responsible for SSI in our community and analyse the microflora of SSI.

\section{Materials and Methods}

Study design: This was a descriptive crosssectional study done for a period of one year (Nov 2012 to Oct 2013) in a tertiary care centre in Bareilly, U.P. All the patients underwent surgeries related to git pathology in the surgery department, either emergency or elective with surgical site infections were included in this study. Patients less than 18, all cases operated else were, or reoperated were excluded from this study.
All traumatic abdominal surgeries were also excluded from this study.

Study procedure: Data regarding patient demography, associated co-morbidities and relevant history were recorded. The diagnosis was made based on clinical examination followed by $\mathrm{X}$-ray abdomen erect, USG abdomen or CECT abdomen. The patient was resuscitated, investigated accordingly, and empirical antibiotics as directed by the surgical team were started. The details of the surgery, the procedure is done, and operative time duration was documented. If any symptom or sign of infection appeared, they had managed accordingly as per standard guidelines. The diagnostic criteria for surgical site diagnosis were as per NSSI guidelines. If any collection of pus was identified, it was drained out and sent for culture and sensitivity test. Antimicrobial susceptibility testing was done by the Kirby-Bauer disk diffusion method. The organisms were tested against the commonly used antibiotics in patients with abdominal surgeries and the susceptibility and resistance patterns available in our region. Growth inhibition zone diameters were measured in millilitres, and results were interpreted as recommended by the Clinical laboratory standards institute (CLSI) guidelines 2013. Minimal inhibitory concentration (MIC) determination was done by Broth microdilution technique and was interpreted as per CLSI guidelines 2013. Follow up was done for 30 days after surgery in patients with surgical site infection and up to 1 year in surgeries with prosthesis.

Statistical analysis: After getting the required information, the collected data were coded, tabulated and analysed. The various statistical techniques, i.e. the mean, standard deviation and test of significance (t-test and chi-square test), were used for drawing valid conclusions. Statistical analysis was done using the student t-test. SPSS 13.0 software was used to calculate the $p$-value. $\mathrm{P}<0.05$ was taken as statistically. A descriptive analysis was done on all variables to obtain a frequency distribution. The mean + S.D. and ranges were calculated for quantitative variables. The Student t-test compared continuous variables. Proportions were analysed with the chi-square test.

\section{Results}

One thousand two hundred fifty abdominal surgeries were included in this study over one year, out of which 870 were elective and 380 emergency 
Surgeries. SSI was associated with $82(9.4 \%)$ cases among elective and $80(21 \%)$ in emergency surgeries. Superficial incision SSI was found to be most common (90 cases), followed by deep incisional SSI (60 cases) and organ/space SSI (12 cases). The patients in the elective surgery group ranged from 18 years to 74 years, with a mean of 42 years. Of these, 545(43.6\%) were females, and $705(56.4 \%)$ were males. In the emergency surgery group, $45(56.25 \%)$ were males, and $35(53.75 \%)$ were females with a mean age of 38 years. SSI was commonest in patients with more than 60 years $(18 \%)$ and least in the $18-40$ age group (12\%). (Table 1) However, these differences were not statistically significant. Co-morbidities associated with these patients operated either elective or emergency are Diabetes mellitus ( $15 \%$ vs $10 \%)$, COPD ( $18 \%$ vs $25 \%$ ), Hypoalbuminemia ( $8 \%$ vs $28 \%$ ) anemia (2\% vs $15 \%)$. Smoking was commonly associated with males in the elective and emergency surgery group (25\% vs $30 \%)$. (Table1)The surgical procedure most commonly associated with SSI in this study was done for small intestine pathology, and the most common indication was ileal perforation peritonitis. The number of cases associated with SSS in this group was $24.6 \%$, followed by gastric/ duodenal (21.6\%). The Least SSI rate was found in hernioplasty. The most common surgery done in this study was cholecystectomy followed by urological surgeries, ventral and inguinal hernias and perforation peritonitis. (Table2)

Out of 162 SSI, the microbial culture report was sterile in 53 cases $(32.7 \%)$. The microbiological organism most commonly isolated were E. Coli $(41.2 \%)$ followed by Staphylococcus aureus $(27.5 \%)$, Klebsiella $(16.5 \%)$, group A betahemolytic streptococci $(9.1 \%)$ and Pseudomonas (5.5\%). (Table.3) E.Coli was found as the most typical organism (45 among 109 cases), causing $41.28 \%$ of the surgical site infections. Staphylococcus Aureus were the second most common organism (30 among 109 patients) accounted for $27.5 \%$ of the infections, followed by Klebsiella in $16.5 \%$, Streptococci in $9.1 \%$ and Pseudomonas in $5.5 \%$. Escherichia coli were most sensitive to Imepenam followed by piperacillin + Tazobactum while sensitive to Ciprofloxacin was $40 \%$, Ceftriaxone $71.1 \%$ and amikacin $73.3 \%$. The sensitivity of Staphylococcus for ampicillin was found to be only $10 \%$, while cephalosporins were found to be more sensitive than aminoglycosides and fluoroquinolones.
Resistance against Cephalosporins was also reported to be less for Klebsiella, Streptococcus and Pseudomonas. Maximum sensitivity was reported for Imepenam and Piperacillin + Tazobactum for all the bacteria cultured in this study. (Table.3)

\section{Table 1: Risk Factors Of SSI}

\begin{tabular}{|c|c|}
\hline Risk Factors associated with SSI & Number of SSI (\%) \\
\hline \multicolumn{2}{|l|}{ Sex } \\
\hline Male & 11.27 \\
\hline Female & 9.5 \\
\hline \multicolumn{2}{|l|}{ Age(Years) } \\
\hline $18-40$ & 12 \\
\hline $40-60$ & 16 \\
\hline$>60$ & 18 \\
\hline \multicolumn{2}{|l|}{ Co-Morbidities } \\
\hline Diabetes Mellitus & 12 \\
\hline Copd & 08 \\
\hline Hypoalbuminemia & 14 \\
\hline Smoking & 10 \\
\hline Anemia & 08 \\
\hline \multicolumn{2}{|l|}{ Type Of Wound } \\
\hline Clean & 3.5 \\
\hline Clean Contaminated & 10.5 \\
\hline Contaminated & 16.4 \\
\hline Dirty & 34 \\
\hline \multicolumn{2}{|l|}{ Type Of Incision } \\
\hline Midline Vertical Incision Around Umbilicus & 25 \\
\hline Upper Midline & 10 \\
\hline Lower Midline & 20 \\
\hline Mac Burneys / Rutherford Morris & 15 \\
\hline Inguinal & 02 \\
\hline Subcostal & 05 \\
\hline Various Incisions For Renal/ Ureteric Surgery & 12 \\
\hline \multicolumn{2}{|l|}{ Duration Of Surgery } \\
\hline Less Than 2 Hours & 14 \\
\hline More Than 2 Hours & 16 \\
\hline
\end{tabular}

Table 2. Surgical procedure and rate of SSI

\begin{tabular}{|l|l|}
\hline \multicolumn{1}{|c|}{ Surgical Procedure } & \multicolumn{1}{c|}{ Number of SSI (N=162) } \\
\hline Gastric/ Duodenal & $35(21.6 \%)$ \\
\hline Small intestine & $40(24.6 \%)$ \\
\hline Appendicectomy & $17(10.4 \%)$ \\
\hline cholecystectomy/ CBD exploration & $20(12.3 \%)$ \\
\hline Hernioplasty & $05(3 \%)$ \\
\hline renal/ ureteric/ bladder stone surgeries & $20(12.3 \%)$ \\
\hline open prostatectomy & $12(7.3 \%)$ \\
\hline Other surgeries & $13(8 \%)$ \\
\hline
\end{tabular}

Table 3: antibiotic sensitivity pattern of the cultured microorganisms 


\begin{tabular}{|c|c|c|c|c|c|}
\hline Sensitivity to Drugs (\%) & E. Coli $(n=45)$ & Staphylococcus aureus $(n=30)$ & Klebsiella $(n=18)$ & Steptococci $(\mathrm{n}=10)$ & Psudomonas $(n=06)$ \\
\hline Ampicillin & 04 & 10 & 05.5 & 80 & 16.6 \\
\hline Amoxicillin + Clavulinic acid & 6.6 & 13.3 & 11.1 & 90 & 16.6 \\
\hline Ciprofloxacin & 40 & 60 & 61.1 & 70 & 66.6 \\
\hline Ceftriaxone & 71.1 & 83.3 & 66.6 & 90 & 66.6 \\
\hline Ceftazidime & 84.4 & 86.6 & 72.2 & 90 & 83.3 \\
\hline Gentamycin & 62.2 & 46.6 & 55.5 & 50 & 66.6 \\
\hline Amikacin & 73.3 & 60 & 72.2 & 80 & 83.3 \\
\hline Piperacillin-Tazobactam & 95.5 & 96.6 & 83.3 & 90 & 83.3 \\
\hline Imipenem & 100 & 100 & 100 & 100 & 100 \\
\hline
\end{tabular}

\section{Discussion}

The incidence of SSI varies from surgeon to surgeon, from hospital to hospital, from one surgical procedure to another and most importantly, from one patient to another for the same procedure[4]. SSI remains one of the most critical factors responsible for the more extended stay of the patient at the hospital, increasing the burden of cost, additional surgical procedures and significant mental trauma to the patient. As in most studies in India, the incidence of SSI in our study was also high at $9.4 \%$ in elective and $21 \%$ in emergency surgeries. The high incidence of SSI in developing countries like India might be due to lack of modular O.T. due to high cost, inadequate postoperative care, poor aseptic environment and sterility during surgical procedures, inadequate infection control and lack of proper surveillance by authorities. In this study, wound class, anaemia, low serum albumin, and hypoxia were significantly associated with surgical site infection.

Surgical wound classification has long been established as an important predictor of postoperative surgical site infections. According to the degree of contamination, wounds may be classified as clean, clean/ contaminated, contaminated and dirty.[5] The incidence of infection increases from $1-5.4 \%$ in clean to $12.8 \%$ in dirty wounds. The risk of infection is highest among colorectal surgeries (9-25\%) [6-8]. In our study, the risk of SSI was higher in dirty and contaminated wounds than in clean and cleancontaminated wounds. Emergency surgeries were associated with a higher SSI rate than elective surgeries in this study. The present study showed an SSI rate of $9.4 \%$ for elective surgeries and $21 \%$ for emergency surgeries. Among the three types, superficial incision SSI was most prevalent (90 cases), followed by deep incisional SSI (60 cases) and finally by organ/space SSI (12 cases).
Exploratory laparotomy (36 \%) was the procedure most commonly associated with SSI in this study, while hernioplasy was least associated with SSI. The risk of SSI is multi-factorial and not entirely determined by the degree of contamination. These are broadly classified as patient factors, operative and environmental characteristics. Patient factors include extremes of age, obesity, tobacco use and smoking, malnutrition, diabetes mellitus, length of preoperative hospitalisation, altered immune response, preoperative nares colonisation with Staphylococcus aureus and concurrent infection in a remote body site. Operative factors include preoperative antiseptic showering, hair removal, skin preparation, antibiotic prophylaxis, duration of surgery, use of foreign prosthesis and drain, level of hemostasis, tissue trauma and suture material used. Apart from that, routine cleaning of surfaces, ventilation in the room, sterilisation of the instruments and drapes also influences the rate of SSI. Many factors might not have been revealed till now. Even the health care personnel can be a source of infection. CDC observed an SSI outbreak of 62 patients operated in seven different hospitals and found the extrinsic contamination of the propofol by the anesthesia personnel was the cause of SSI. The important factors in these outbreaks were multiple uses of a single vial for several patients and improper aseptic technique. $[9,10]$.

The microbiological organism most commonly isolated were E. Coli $(41.2 \%)$, followed by Staphylococcus aureus (27.5\%), Klebsiella (16.5\%), group A beta-hemolytic streptococci (9.1\%) and Pseudomonas(5.5\%). In many studies, it has been documented that the responsible microflora originates from the patient's endogenous flora. The organism isolated from the wound depends on the type of surgery. The most commonly isolated bacteria were Staphylococcus aureus, coagulasenegative Staphylococci, Enterococcus and E. coli. [3]. 
In clean surgical procedures, Staphylococcus aureus was most commonly isolated from cases with SSI, which is usually residing in the exogenous environment or the patient's skin flora. SSI associated with clean-contaminated, contaminated and dirty were usually polymicrobial in origin and closely resembling the normal endogenous microflora of the surgically resected organ. [11]. It has also been reported by the National Nosocomial Infections Surveillance System (NNIS) that the microflora of SSI has not shifted significantly in the last decade. [1] In few studies, the predominant isolate of SSI was Klebsiella pneumoniae, followed by Staphylococcus aureus, E. coli, and Pseudomonas spp. Klebsiella pneumonia is also natural habitat in the gastrointestinal tract as $E$. Coli. In their study, most of the operations performed were laparotomies, and most wounds were either clean-contaminated, contaminated or dirty with spillage from the GIT content. Most SSIs were caused by multi-drug resistant (MDR) organisms[12]. Duration of operation time was not significantly associated with SSI in this study. Previous studies have reported the duration of surgery as a risk factor for surgical site infections $[13,14]$.

\section{Conclusion}

Although prolonged operative time leads to fatigue awareness and practice of aseptic measures along with advancement in surgical technique, improvement in the operative environment has improved the surgical outcome.

\section{What this study adds to existing knowledge}

Surgical site infection still causes considerable morbidity and high cost to the health care system and is becoming increasingly crucial in medico-legal aspects. Prevention of SSI requires a targeted approach to improve health care workers' knowledge, practising the guidelines strictly regarding asepsis and optimising the patient before surgery. Reduction in rate of SSI will improve cosmesis and make the results of operations better as a whole.

\section{Contribution by different authors}

Dr Atul Kumar: Concept and data collection
Dr Shivani Sinha: Manuscript preparation and final drafting of the paper

\section{Reference}

01. National Nosocomial infection Survillance (NNIS) System. National Nosocomial infection Surveillance (NNIS) report, data summary from October 1986April 1996, issued May 1996, A report from the National Nosocomial infection Surveillance (NNIS) System. Am J Infect Control. 1996 Oct. 24(5):380-8 [Crossref][PubMed][Google Scholar]

02. Ganguly P S, Md Yunus Khan, and Abida Malik. Nosocomial infections and hospital procedures. Indian Journal of Community Medicine. 25;1(2000):39. [Crossref][PubMed][Google Scholar]

03. Owens $C D$, Stoessel K. Surgical site infections: epidemiology, microbiology and prevention. J Hosp Infect. 2008 Nov;70 Suppl 2:3-10. doi: 10.1016/S0195-6701(08)60017-1

[PubMed][Google Scholar]

[Crossref]

04. Nichols RL. Preventing surgical site infections: a surgeon's perspective. Emerg Infect Dis. 2001 MarApr;7(2):220-4. doi: 10.3201/eid0702.010214 [Crossref][PubMed][Google Scholar]

05. Martone WJ, Nichols RL. Recognition, prevention, surveillance, and management of surgical site infections: introduction to the problem and symposium overview. Clin Infect Dis. 2001 Sep 1;33 Suppl 2:S67-8. doi: 10.1086/321859 [Crossref][PubMed][Google Scholar]

06. Kobayashi M, Mohri Y, Inoue Y, Okita Y, Miki C, Kusunoki M. Continuous follow-up of surgical site infections for 30 days after colorectal surgery. World journal of surgery. 32;6(2008): 1142-1146. [Crossref][PubMed][Google Scholar]

07. Blumetti J, Luu M, Sarosi G, Hartless K, McFarlin J, et al. Surgical site infections after colorectal surgery: do risk factors vary depending on the type of infection considered?. Surgery. 142;5(2007):704711. [Crossref][PubMed][Google Scholar]

08. Konishi T, Watanabe T, Kishimoto J, Nagawa $\mathrm{H}$. Elective colon and rectal surgery differ in risk factors for wound infection: results of prospective surveillance. Annals of surgery. 244;5(2006):758. [Crossref][PubMed][Google Scholar] 
09. Bennett SN, McNeil MM, Bland LA, Arduino MJ, Villarino $M E$, Perrotta DM, et al. Postoperative infections traced to contamination of an intravenous anesthetic, propofol. N Engl J Med. 1995 Jul 20;333(3): 147-54. doi:

10.1056/NEJM199507203330303

[Crossref]

[PubMed][Google Scholar]

10. Nichols RL, Smith JW. Bacterial contamination of an anesthetic agent. N Engl J Med. 1995 Jul $20 ; 333(3): 184-5$.

doi:

10.1056/NEJM199507203330310

[Crossref]

[PubMed][Google Scholar]

11. Nichols RL. Prevention of infection in high risk gastrointestinal surgery. Am J Med. 1984 May 15;76(5A):111-9. doi: 10.1016/00029343(84)90252-3 [Crossref][PubMed][Google Scholar]
12. Lubega $A$, Joel $B$, Justina Lucy $N$. Incidence and Etiology of Surgical Site Infections among Emergency Postoperative Patients in Mbarara Regional Referral Hospital, South Western Uganda. Surg Res Pract. 2017;2017:6365172. doi: 10.1155/2017/6365172 [Crossref][PubMed][Google Scholar]

13. Giri S, Kandel BP, Pant S, Lakhey PJ, Singh YP, Vaidya P. Risk factors for surgical site infections in abdominal surgery: a study in Nepal. Surg Infect (Larchmt). 2013 Jun;14(3):313-8. doi: 10.1089/sur.2012.108 [Crossref][PubMed][Google Scholar]

14. Ikeanyi UO, Chukwuka CN, Chukwuanukwu TO. Risk factors for surgical site infections following clean orthopaedic operations. Niger J Clin Pract. 2013 Oct-Dec;16(4):443-7. doi: 10.4103/11193077.116886 [Crossref][PubMed][Google Scholar] 